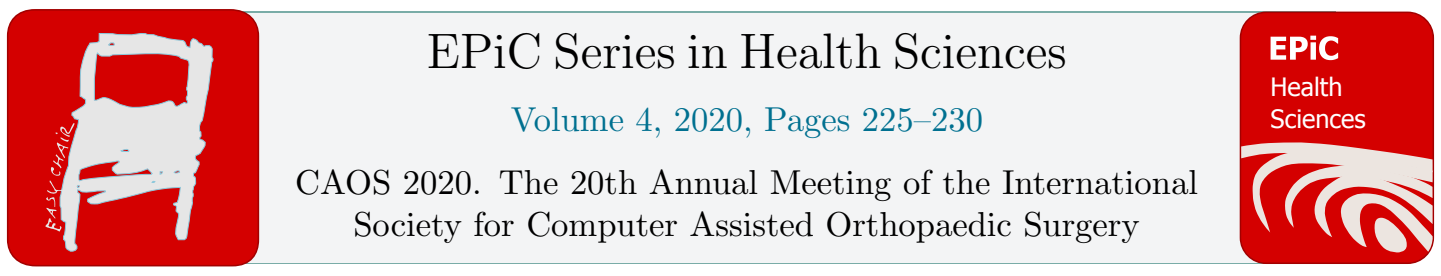

\title{
Patients Specific Spine Simulators for Surgical Training and Rehearsal in Pedicule Screws Placement: a New Way for Surgical Education
}

\author{
Parchi $\mathrm{P}^{1}$, Carbone $\mathrm{M}^{2}$, Condino $\mathrm{S}^{2}, \mathrm{~S} \mathrm{Stagnari}^{1}$, D Rocchi ${ }^{1}$, Colangeli $\mathrm{S}^{3}$, \\ Ferrari $\mathrm{M}^{2}$, Scaglione $\mathrm{M}^{1}$, Ferrari $\mathrm{V}^{2}$ \\ ${ }^{1}$ Ist Orthopedic Division University of Pisa, Italy \\ ${ }^{2}$ Endocas Center University di Pisa, Italy \\ ${ }^{3}$ 2nd Orthopedic Division University of Pisa, Italy \\ paolo.parchi@unipi.it, marina.carbone@endocas.org, sara.condino@endocas.org
}

\begin{abstract}
In pedicle screws placement using a free-hand technique or a fluoroscopic guided technique the main difficulties are facing to the bone morphology (i.e in deformity cases) and it could be easily reproduced in a patient's specific spine simulator (we can choose the case). The aim of this work is to evaluate the use of 3D printed patientspecific models (3D printing) not only as a surgical planning tool but also as a surgical training tool in spine surgery and in particular in pedicle screws placement. The manufacturing of patient-specific physical replica involves the elaboration of CT dataset and rapid prototyping techniques. . Five resident surgeons were involved in different training sessions on simulators. To evaluate the exact screws position we performed a CT evaluation of each instrumented simulators. Statistical analysis was conducted using SPSS software. A total of 120 pedicle screws were positioned, 90 screws were well-positioned and 30 screws were bad-positioned. There were a significant difference $(\mathrm{p}=0.000008)$ between the bad-positioning screw rate of the "senior" resident (13/72) and those of "young" participants (17/48). Timeline analysis of pedicle instrumentation training showed the presence of a learning effect, with a lower error rate in the latest session $(\mathrm{p}=000001)$. We believe that the use of patient-
\end{abstract}


specific surgical simulators, especially for those surgical tasks in which the complexity is mainly linked to the spine morphology (i.e. deformity), may represent a valid alternative to the use of cadavers that generally present a standard or otherwise poorly predictable anatomy.

KEYWORDS: 3d printing, spine surgery, pedicular screws, surgical training

\section{INTRODUCTION}

Pedicle screws fixation to stabilize spine fusion is gold standard amongst posterior instrumentation techniques. Currently, pedicle screws are positioned using a free-hand technique or under fluoroscopic guidance with a variable risk of screw misplacement depending on several factors such as the presence of spine deformity and the skill of the surgeons with a high the error rate reported literature (10-40\%).

In the last year in orthopedic and spine surgery there was an increasing interest in the development of surgical simulators using methods of additive manufacturing combined or not with augmented reality systems (hybrid simulators) [1-4]. In pedicle screws placement using a free-hand technique or a fluoroscopic guided technique the main difficulties are facing to the bone morphology (i.e in deformity cases) and it could be easily reproduced in a patient's specific spine simulator (we can choose the case) while it is very difficult to find in a cadaver and it is also not predictable.

The aim of this work is to evaluate the use of 3D printed patient-specific models (3D printing) not only as a surgical planning tool but also as a surgical training tool in spine surgery and in particular in pedicle screws placement.

\section{METHODS}

The manufacturing of patient-specific physical replica involves the elaboration of CT dataset and rapid prototyping techniques as described in previous works [1-3]. The stack of CT images in DICOM format is processed using a semi-automatic tool, the EndoCAS Segmentation Pipeline integrated in the open source software ITK-SNAP 1.5 [34], to generate the 3D virtual models of the patient bone structures. Then mesh optimization stages are performed via the open source software MeshLab A 3D printer (Dimension Elite Stratasys) is used to turn the 3D virtual models into tangible 3D synthetic replicas made of acrylonitrile butadiene styrene (ABS). ABS is commonly used for the manufacturing of bone replica for orthopedic surgery simulation, since it sufficiently replicates the mechanical behavior of the natural tissue, and it is compatible with X-Ray examinations. Intersomatic disks were obtained by cutting out 
a sheet of silicone RTV (Room-Temperature-Vulcanizing) ECOFLEX (bicomponent silicone consisting of a base and a catalyst in a 1:1 ratio). Cut-out discs were then modeled on each clinical case to replicate the heights of intersomatic discs of each level. RTV ECOFLEX was also indicated for its radiolucency to fluoroscopic imaging. Once the disks are positioned, the structure is stabilized with common RUBBER BANDS and with RTV DRAGONSKIN FX-PRO 67 silicone casting on the front surface of vertebral bodies and the respective disks, mimicking anterior longitudinal ligament. 3D printed vertebrae maintained a mobility similar to that found in vivo. Finally, bone replicas are embedded in a soft synthetic polyurethane foam FlexFoamIT TM III (via casting technique) which represent paravertebral soft tissues during the simulation procedure, and they are covered with an RTV silicone based skin-like layer Ecoflex skin coating, to allow an accurate simulation of the bony anatomical landmarks palpation and surgical incision [Fig.1].
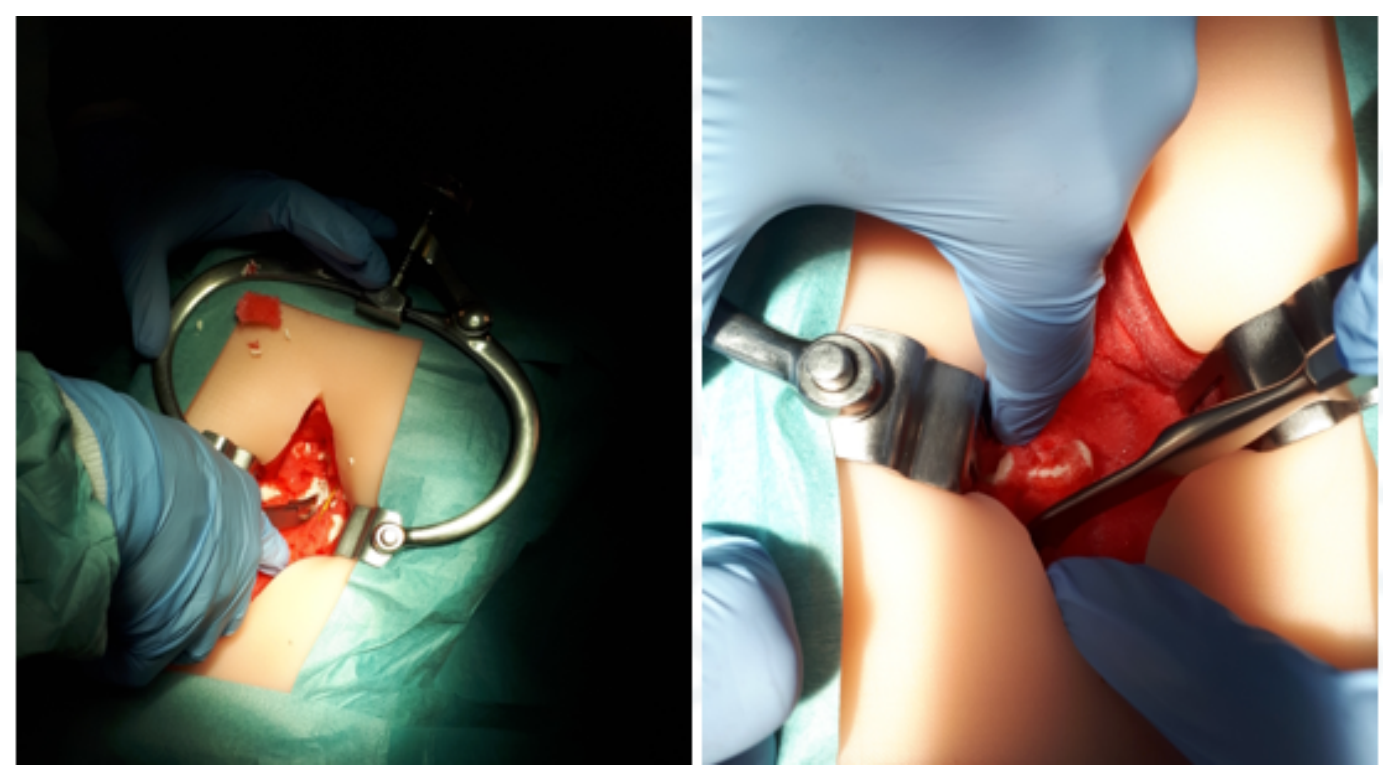

Fig. 1

Five resident surgeons were involved in different training sessions on simulators [Fig. 2]. Before starting the simulation procedure the experienced surgeon explained the different phases of the free-hand technique for pedicle screws placement. At the end of each procedure we performed a x-ray evaluation of the screw position in order to discuss (debrifing) evident errors in screws placement. To evaluate the exact screws position we performed a CT evaluation of each instrumented simulators. The length 
of time in completing procedures was also recorded. Statistical analysis was conducted using SPSS software.

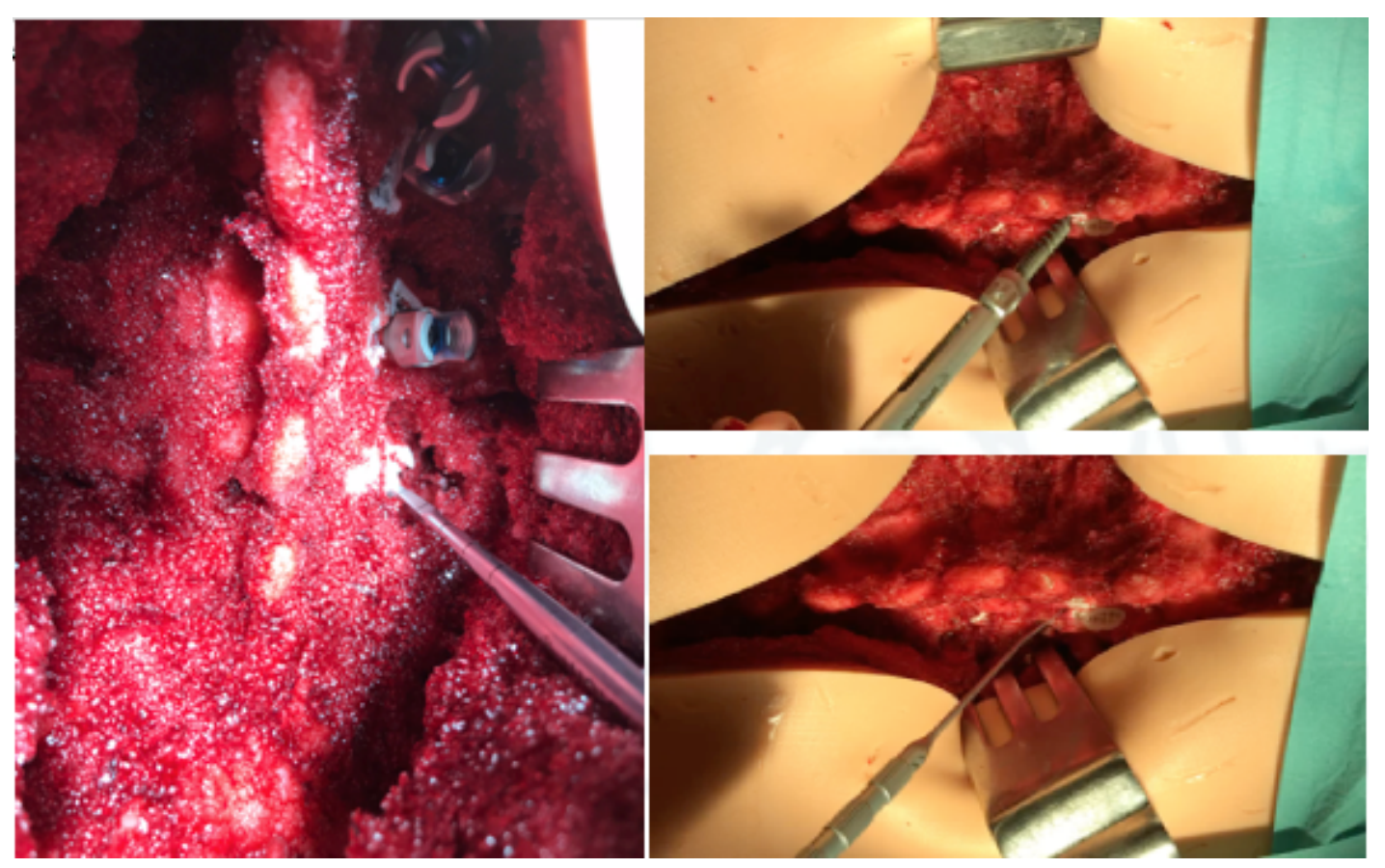

Fig. 2

\section{RESULTS}

A total of 120 pedicle screws were positioned. CT acquisitions of 3D printed simulators were used to classify the degree of violation of peduncular wall. According to this classification, 63 screws were in " $A$ " category (No pedicle wall violation), 27 screws in "B" category (wall violation $<2 \mathrm{~mm}$ ), 16 screws in "C" category (wall violation $>2 \mathrm{~mm},<4 \mathrm{~mm}$ ), 14 screws in "D" category (wall violations $>4 \mathrm{~mm}$ ). A total of 90 screws were well-positioned ("A" category + "B" category) and 30 screws were bad-positioned ("C" category + "D" category). Screws were positioned in three subsequent temporal sessions, of which the first two under the supervision of an experienced surgeon while the last one, without a teacher's help. There were a significant difference $(\mathrm{p}=0.000008)$ between the bad-positioning screw rate of the "senior" resident (13/72) and those of "young" participants (17/48). This result is significant $(\mathrm{p}=0.000008)$.

Timeline analysis of pedicle instrumentation training showed the presence of a learning effect, with a lower error rate in the latest session $(p=000001)$. Also, the 
average time required to complete five-level instrumentation (L1-L5) was from 60 min to $30 \mathrm{~min}$ in the last session. In addition, the average time needed by senior residents for single screw positioning was lower than those found in most inexperienced members. Each of the participants emphasized the realism of these simulators, especially regarding perceived differences with surgical instrumentation between cortical and trabecular bone.

\section{DISCUSSION}

Only a few studies have been reported in the literature to evaluate the effectiveness of surgical training on specific patient simulators $(4,5)$. Park et al. proposed a study to evaluate the educational effects of 3D printed life-size spinal training for inexperienced surgeons, reporting the existence of a "learning effect" during the repetition of surgical procedures on subsequent models, with a progressive increase in the accuracy of pedicle screws position and decrease of peduncolar infractions (6). The use of $3 \mathrm{D}$ printing to reproduce spinal segments can be a valid tool for surgical training of young practitioners, demonstrating a learning effect on spinal instrumentation technique with pedicle screws and suggesting the establishment of additional training courses in the educational path of young doctors. The solid model of the spine can faithfully reproduce the surgical complexity of the patient and it allows to generate surgical simulators with an increasing difficulty to perform structured training paths: from the "simple" case to the "complex" case.

The cost of each simulator (500-700€) appears to be similar to that of cadaveric training, but there are further advantages to consider: the possibility of recovering surgical instrumentation (including screws) at the end of each session and the absence of costs relating to sterilization procedures.

We believe that the use of patient-specific surgical simulators, especially for those surgical tasks in which the complexity is mainly linked to the spine morphology (i.e. deformity), may represent a valid alternative to the use of cadavers that generally present a standard or otherwise poorly predictable anatomy. 


\section{References}

1 P. Parchi, S. Condino, M. Carbone, M. Gesi, V. Ferrari, M. Ferrari, \& M. Lisanti, Total hip replacement simulators with virtual planning and physical replica for surgical training and reharsal, Proceedings of the 12th IASTED International Conference on Biomedical Engineering, BioMed 2016, 2016, 97-101

2 P.D. Parchi, V. Ferrari, N. Piolanti, L. Andreani, S. Condino, G. Evangelisti, \& M. Lisanti, Computer tomography prototyping and virtual procedure simulation in difficult cases of hip replacement surgery, Surg Technol Int, 232013, 228-234. istep:

3 S. Condino, G. Turini, P.D. Parchi, R.M. Viglialoro, N. Piolanti, M. Gesi, M. Ferrari, \& V. Ferrari, How to Build a Patient-Specific Hybrid Simulator for Orthopaedic Open Surgery:

Benefits and Limits of Mixed- Reality Using the Microsoft HoloLens, Journal of Healthcare Engineering, 2018.

4 K. Atesok, J.D. Mabrey, L.M. Jazrawi, \& K.A. Egol, Surgical simulation in orthopaedic skills training, J Am Acad Orthop Surg, 20(7), 2012, 410-422.

5 W.H.A. Ryu, N. Dharampal, A.E. Mostafa, E. Sharlin, G. Kopp, W.B. Jacobs, R.J. Hurlbert, S. Chan, \& G.R. Sutherland, Systematic Review of Patient-Specific Surgical Simulation: Toward Advancing Medical Education, J Surg Educ, 74(6), 2017, 1028-1038.

6 Park, H. J., Wang, C., Choi, K. H. \& Kim, H. N. Use of a life-size three-dimensional-printed spine model for pedicle screw instrumentation training. J. Orthop. Surg. Res. 13, 86 (2018) 